\title{
Modos discontinuos y erráticos de transitar un hospital especializado en salud mental y adicciones de la Ciudad de Buenos Aires: la mirada de profesionales de la salud
}

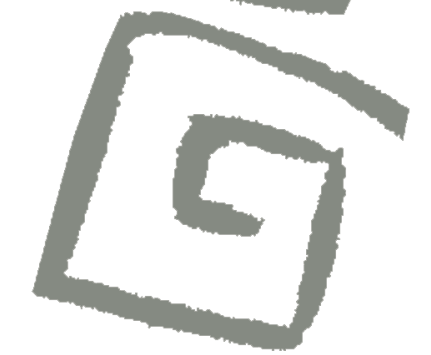

${ }^{1}$ Autora de correspondencia. Licenciada en Psicología. Psicóloga, Centro de Especialidades Médicas de Referencia $N^{\circ} 2$, Gobierno de la Ciudad Autónoma de Buenos Aires, Ciudad Autónoma de Buenos Aires, Argentina. $\triangle$ (iD)

${ }^{2}$ Licenciada en Psicología. Psicóloga, Centros de Salud y Acción Comunitaria $N^{\circ}$ 31, Gobierno de la Ciudad de Buenos Aires, Ciudad Autónoma de Buenos Aires, Argentina. $\triangle$ iD

${ }^{3}$ Licenciada en Psicología Instructora de residentes, Residencia de Salud Mental, Gobierno de la Ciudad de Buenos Aires, Ciudad Autónoma de Buenos Aires, Argentina. $\bowtie$ (iD)

${ }^{4}$ Magíster en Estudios de Género. Psicóloga, Secretaría de Género y Diversidad Sexual, Ministerio Público de la Defensa, Ciudad Autónoma de Buenos Aires, Argentina. $\triangle$ (iD)

\author{
Discontinuous and erratic ways of passing through a hospital \\ specialized in mental health and addictions in the City of \\ Buenos Aires: the point of view of the health professionals
}

Ana Alli ${ }^{1}$, Jesica Minond ${ }^{2}$, Yesica Embil' ${ }^{3}$, Malena Kiss Rouan ${ }^{4}$

RESUMEN El objetivo fue describir la perspectiva de las y los profesionales de la salud sobre los recorridos clínicos erráticos de personas con consumos problemáticos de sustancias que asistían a un hospital monovalente de la Ciudad de Buenos Aires, especializado en salud mental y adicciones. Entre octubre de 2017 y octubre de 2018, se realizó una investigación cualitativa de carácter exploratorio, basada en seis estudios de casos que se reponen desde una investigación previa. Para ello, se trabajó con la lectura y el análisis de historias clínicas y entrevistas semiestructuradas a las y los profesionales intervinientes. Entre los principales resultados se encontró que la definición de recorrido clínico errático resultaba poco descriptiva del recorrido de algunas personas usuarias de los dispositivos, por lo que se apeló a un nuevo concepto: el tramo. Esta noción recupera el momento en que la trayectoria se sostiene, aunque implique el pasaje por un dispositivo diferente. Con relación a los objetivos y las estrategias terapéuticas, se relevó una notoria dificultad, tanto para nombrarlos como para delimitarlos. Finalmente resultó Ilamativa la poca sistematización de las derivaciones intrahospitalarias entre equipos tratantes de salud mental, en comparación a las interconsultas médicas clínicas y derivaciones a otros efectores de salud.

PALABRAS CLAVES Personal de Salud; Servicios de Salud; Usuarios de Drogas; Argentina.

\begin{abstract}
The purpose of this study was to describe the perspective of health professionals regarding the discontinuous and erratic clinical trajectories of individuals with problematic substance use at a hospital specialized in mental health and addiction in the City of Buenos Aires. From October 2017 to October 2018, a qualitative exploratory study was carried out based on six case studies recovered from previous research. Medical records were analyzed and semi-structured interviews were conducted with the professionals involved. Among the principal results, we found that the definition of erratic clinical trajectories formulated was not very descriptive of the course of some cases, leading us to propose a new concept, that of the segment (el tramo). This concept accounts for the moment in which the clinical trajectory is sustained, even if that entails switching to a different health service. With respect to objectives and therapeutic strategies, we found a clear difficulty on the part of professionals to name and describe them. Lastly, we found alarmingly little systematization of in-hospital referrals among mental health treatment teams, compared to clinical medical consultations and referrals to other health care providers.
\end{abstract}

KEY WORDS Health Personnel; Health Services; Drug Users; Argentina. 


\section{INTRODUCCIÓN}

Dentro del campo del consumo problemático de sustancias, la cuestión de la discontinuidad en los tratamientos resulta una problemática relevante para el equipo de profesionales de la salud. Los estudios sobre el tema ${ }^{(1)}$ muestran que, de acuerdo a la percepción del personal de salud, al menos la mitad de las personas que consultan no siguen las indicaciones de los equipos $(59,3 \%$ ) y/o abandonan los tratamientos $(46,8 \%)$. Sin embargo, la complejidad de la problemática no se agota en las conductas individuales de las personas que acuden al hospital, sino que es necesario incorporar al análisis las estrategias terapéuticas ofertadas por los servicios de salud, los cambios en la legislación y la coyuntura sociopolítica.

Al mismo tiempo, para analizar los fenómenos de las adicciones es necesario tener en cuenta los tipos subjetivos constituidos en la sociedad actual. Según Lewkowicz ${ }^{(2)}$, la adicción se tornó una amenaza para el individuo, en el sentido de que constantemente se puede caer en ella. Para el autor, la adicción existe como categoría identitaria porque socialmente es posible la subjetividad adictiva. Oriol Romaní ${ }^{(3,4)}$ define las adicciones como un fenómeno producto de cambios sociales, culturales y tecnológicos. Es un conjunto de procesos a través de los cuales se expresan ciertos malestares más o menos graves, que pueden tener causas diversas, pero cuyo problema principal sería la organización del conjunto de la vida cotidiana de un individuo alrededor del consumo, más bien compulsivo, de determinadas sustancias.

Esta investigación se inscribió en la perspectiva de la salud colectiva ${ }^{(5,6,7)}$, los estudios sobre adicciones ${ }^{(1,2,3,4)} y$, dentro de los paradigmas existentes para el abordaje de las adicciones, se ha tomado el modelo de reducción de riesgos y daños, que permite analizar el fenómeno por fuera de la lógica de curar un síntoma o una enfermedad. Dentro de este paradigma, un tratamiento tiene por finalidad acompañar las decisiones de una persona, incluso si decidiera continuar consumiendo, para orientarlas hacia estrategias de salud y cuidado, tanto dentro del sistema de salud como en otras organizaciones de la comunidad, y permite flexibilizar el encuadre disminuyendo el umbral de exigencia para acceder a un tratamiento.

El concepto de adherencia al tratamiento ha sido investigado como un fenómeno central del proceso y gestión de la enfermedad. Tradicionalmente fue entendido como todas las acciones que un individuo realiza con el fin de mejorar su estado de salud, siguiendo las recomendaciones y prescripciones profesionales. En este sentido, nos interesa incluir el concepto de proceso de gestión de la enfermedad, el cual introduce la posibilidad de pensar la adherencia en relación con las trayectorias de vida de un sujeto; trayectorias atravesadas por el trabajo subjetivo de una persona ante el requerimiento de tratamiento, las demandas familiares, laborales y económicas, las estigmatizaciones asociadas a la enfermedad, las condiciones de vida usualmente caracterizadas por la pobreza, la desocupación, la precariedad laboral o habitacional y las dificultades en la accesibilidad, tanto a los servicios de salud como a los recursos sociales $^{(8)}$.

Entendemos accesibilidad como los encuentros y desencuentros entre las personas usuarias y el sistema de salud ${ }^{(6)}$, los cuales permiten analizar las intervenciones que favorecen la adherencia. Desde esta perspectiva se tiene en cuenta, además de la accesibilidad inicial, la dimensión de permanencia de una persona y la dignidad en la atención ${ }^{(9)}$.

Con relación a los tratamientos, se utilizó el concepto de itinerarios terapéuticos ${ }^{(10)}$ que refiere a todos los movimientos desencadenados por un individuo para la recuperación o preservación de la salud. Consiste en una sucesión de acontecimientos y toma de decisiones que, teniendo como objeto el tratamiento de la enfermedad, construye un determinado circuito. Estos movimientos pueden incluir diferentes recursos que engloban desde cuidados caseros y prácticas religiosas hasta dispositivos biomédicos predominantes ${ }^{(11)}$.

La Figura 1 contiene los conceptos centrales utilizados en la investigación, que resultan 


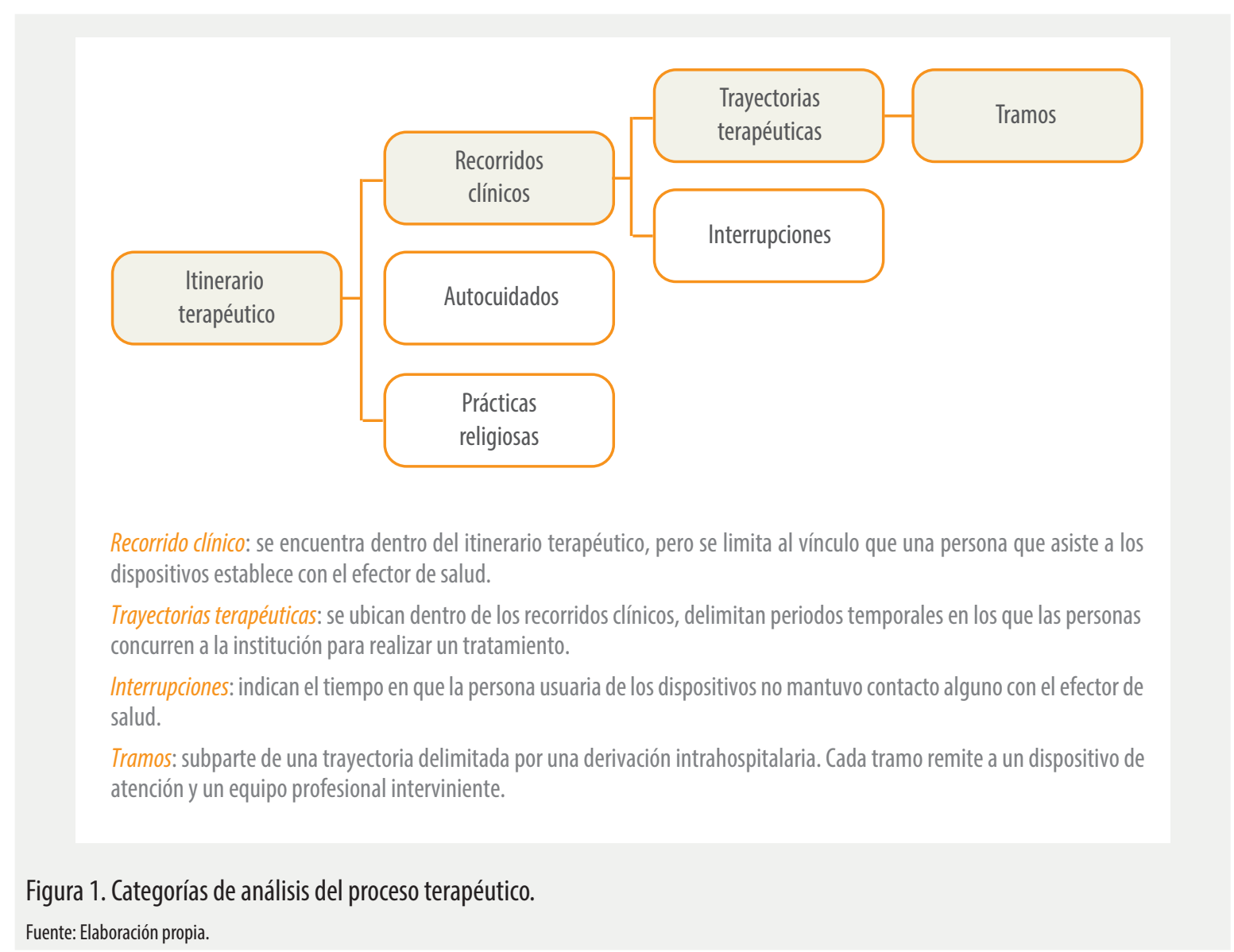

fértiles y necesarios para describir el proceso que analizamos.

Como trabajadoras de la institución nos ha interpelado el modo en que las personas se relacionan con el hospital y, particularmente, con los tratamientos. Observamos que el vínculo es generalmente discontinuo y errático, dado que inician el tratamiento, lo interrumpen, vuelven a consultar en reiteradas ocasiones y transitan por diferentes dispositivos dentro del hospital. Notamos que esta cuestión se repite en los dispositivos del hospital, y es denominado por las y los profesionales como "falta de implicación", "escasa adherencia" o "ausencia de responsabilidad subjetiva".

Este trabajo es la continuación de la investigación "Encuentros y desencuentros entre los usuarixs y los servicios de salud mental", realizada entre junio de 2016 y mayo de 2017 en el marco del programa de formación de la Residencia Interdisciplinaria en Salud Mental (RISAM), que funciona en un hospital mono- valente de la Ciudad Autónoma de Buenos Aires especializado en salud mental y adicciones. A partir de un screening inicial con profesionales de los dispositivos de la institución (informantes claves), se detectó la relevancia del problema, tanto en el interés manifestado por las y los profesionales como en la cantidad de personas que fueron ubicadas dentro del perfil que presentan discontinuidad en los recorridos clínicos. En total, se mencionaron 77 personas usuarias, de las cuales el $31,1 \%$ fue nombrada de manera repetida en dispositivos diferentes.

Para la presente investigación se relevó la totalidad de profesionales intervinientes en seis estudios de caso seleccionados de la investigación anterior y se constituyó una muestra a los fines de indagar las consideraciones respecto al caso clínico, las dificultades y facilitadores en las trayectorias terapéuticas, y las estrategias terapéuticas implementadas, con el objetivo de profundizar la descripción 
del fenómeno, por qué y cómo ocurre, desde la mirada de las y los profesionales.

Apoyamos el supuesto de que el mantenimiento del tratamiento responde a diferentes variables: características psicopatológicas, modo de circulación propio del tipo subjetivo ofrecido por la época, comprensión del fenómeno por parte del equipo profesional, incidencia de los límites/características de la institución, marco normativo(12,13). Consideramos que la tendencia a implementar estrategias terapéuticas flexibles propicia el vínculo terapéutico y apuntala la continuidad del tratamiento. Este trabajo intenta abonar a la construcción de conocimiento sobre las estrategias de abordaje para los tratamientos de consumos problemáticos que favorezcan el cuidado de la salud de las personas usuarios de los dispositivos, además de proponerse como material para delinear nuevas líneas de trabajo e intervención sobre la problemática.

\section{ASPECTOS METODOLÓGICOS}

El presente trabajo siguió los lineamientos de la metodología cualitativa de tipo exploratorio. Se realizaron entrevistas semiestructuradas a las y los profesionales de salud mental intervinientes en los seis casos de estudio seleccionados de la investigación previa, cuyos resultados fueron utilizados como fuente de datos. Se adoptó un enfoque interpretativo, con el propósito de comprender e interpretar la significación de la acción humana ${ }^{(14)}$ a partir del análisis y la descripción de las narrativas del personal de salud entrevistado.

Se relevó el total de equipos profesionales intervinientes en cada uno de los seis recorridos clínicos que componen los casos de referencia. Para la elección de las y los profesionales, se realizó un muestreo por conveniencia en cada trayectoria y se decidió seleccionar dos profesionales por cada recorrido clínico, que sumaron un total de doce referentes claves a quienes entrevistar. Para llevar adelante esta selección, visibilizamos los momentos de cambios relevantes en cada recorrido clínico y qué profesionales acompañaron al usuario en ese período. Se desestimó como criterio de selección la "mayor permanencia" de una persona en una trayectoria, como equivalente a la significatividad en su proceso de salud-enfermedad-atención, aunque se consideró que la mayor cantidad de tiempo referenciado a un equipo podía influir en la información disponible en el recuerdo de las y los profesionales a entrevistar (Tabla 1).

A los fines de evitar un sesgo en el perfil del personal de salud, se decidió elegir en igual cantidad profesionales varones y mujeres de las diferentes disciplinas que conforman los equipos intervinientes (trabajo social, psiquiatría y psicología) en los siguientes dispositivos de atención del hospital:

- Consultorios externos: dispositivo de tratamiento ambulatorio del segundo nivel de atención, que cuenta con profesionales de psicología, psiquiatría, trabajo social, terapia ocupacional y ofrece tratamientos mayormente individuales y consultas familiares.

- Hospital de día: dispositivo de asistencia ambulatoria de mayor complejidad e intensidad como internación diurna, que ofrece tratamientos individuales, grupales y talleres.

- Internación aguda: brinda tratamiento intensivo e integral del episodio agudo, entendido como excitación psicomotriz, descompensación psicótica, consumo compulsivo de sustancias, episodios de urgencia subjetiva, situaciones en las que se objetiva riesgo.

- Internación: dispositivo que recibe a personas con un cuadro clínico que requiere de mayor tiempo de internación.

- Atención a la demanda espontánea: dispositivo "puerta de entrada" a la institución, que realiza el proceso de admisión mediante entrevistas interdisciplinarias (psiquiatría, psicología y/o trabajo social) y cuenta con grupos terapéuticos. Deriva a los diferentes dispositivos del hospital según la necesidad de cada persona.

Todos los equipos de profesionales entrevistados son actualmente trabajadores de la institución, de 30 a 50 años de edad, con una experiencia clínica en el ámbito público de 5 a 10 años. 
Tabla 1. Dispositivos de atención, trayectorias, tramos, profesionales intervinientes y entrevistados, según casos seleccionados. Ciudad Autónoma de Buenos Aires (2016-2017).

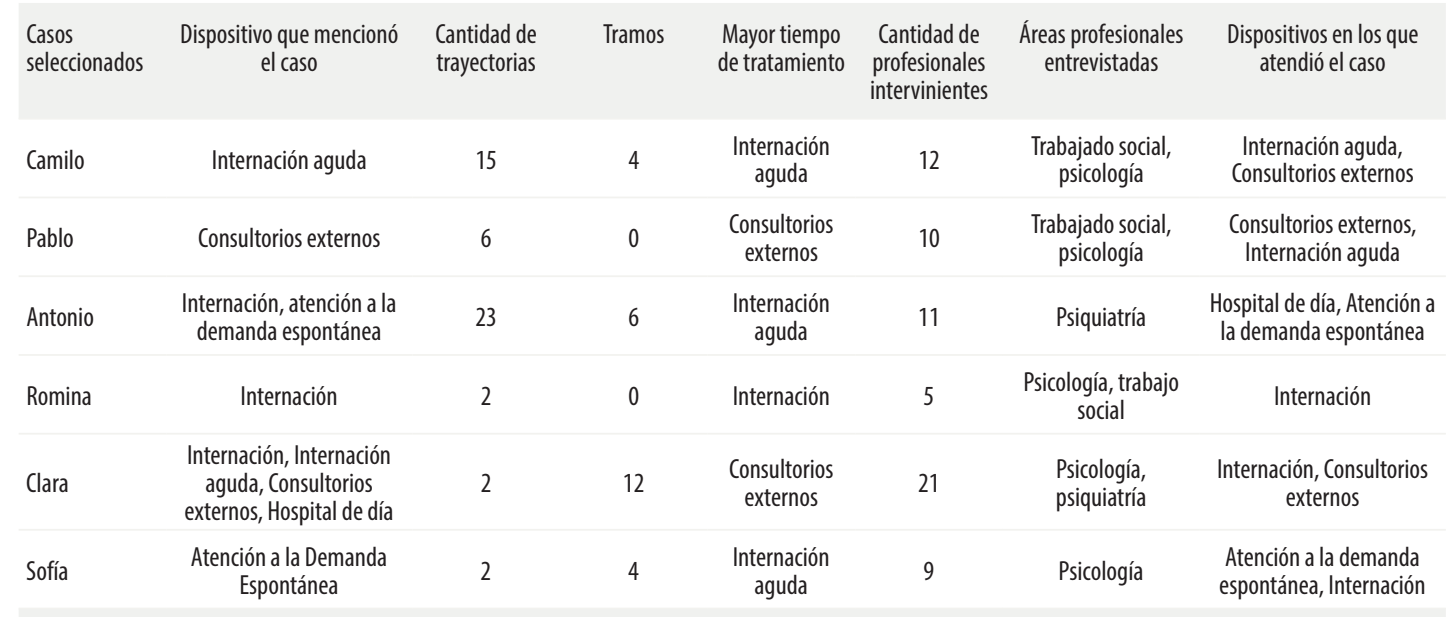

Fuente: Elaboración propia a partir de una selección de datos recolectados en la investigación "Encuentros y desencuentros entre los profesionales y los usuarios", Residencia Interdisciplinaria en Salud Mental (RISAM).

La entrevista estuvo centrada en tres ejes principales que sirvieron luego para el proceso de análisis de los datos:

\section{- Las consideraciones respecto al caso clínico:} caracterización de las personas usuarias de los dispositivos, los equipos profesionales intervinientes, los motivos de consulta, las hipótesis diagnósticas, la función del consumo en el caso singular del usuario, los objetivos del tratamiento y la modalidad del vínculo de cada persona con el equipo profesional interviniente.

- Las dificultades y los facilitadores en las trayectorias terapéuticas: los recursos de las personas que asisten al hospital tomando en consideración sus aspectos económicos, sociales y subjetivos; los recursos facilitados por el efector de salud, el impacto de estos en la trayectoria terapéutica, los obstáculos en el tratamiento, las hipótesis y previsibilidad del abandono o revocación de las personas y la lógica de implementación de las derivaciones.

- Las estrategias terapéuticas: las indicaciones terapéuticas a nivel individual, familiar, grupal, institucional e intersectorial; el lugar otorgado a las decisiones de cada persona en el tratamiento; el trabajo con las pautas de cuidado; las estrategias implementadas en momentos agudos de la trayectoria; la conceptualización respecto al alta del tratamiento y la referencia de cada persona a la institución.

El trabajo de campo fue realizado durante el año 2018. Las entrevistas fueron efectuadas mayoritariamente en la institución durante la jornada laboral de cada profesional y tuvieron una duración promedio de una hora. Se intentó buscar un espacio tranquilo y apartado para no interferir en el desarrollo de las entrevistas y, al mismo tiempo, garantizar la privacidad y la confidencialidad del material. El registro de las entrevistas se realizó a través de notas y grabaciones que fueron luego desgrabadas. A partir de un análisis cualitativo y temático, se ponderaron las estructuras de relevancia de los discursos individuales en función de los conceptos y ejes temáticos relevantes de las entrevistas, en pos de profundizar la descripción de la perspectiva de las y los profesionales sobre los recorridos clínicos de las personas con consumo problemático de sustancias ${ }^{(14)}$.

Con relación a los resguardos éticos, esta investigación se adecuó a los criterios de consentimiento informado y confidencialidad que 
se aplican en los estudios sobre salud. Con el fin de asegurar los derechos de quienes participaron de la investigación y resguardar su identidad, se reemplazaron los nombres reales por nombres ficticios. La investigación fue aprobada por el comité de ética de la institución en la que se realizó el trabajo de campo.

\section{RESULTADOS}

\section{Consideraciones respecto al caso clínico}

El primer eje de la entrevista hizo foco en recabar información sobre la construcción del caso por parte de los equipos profesionales intervinientes. Al hablar de las características generales de las personas usuarias de los dispositivos se observó, en la mayoría de las entrevistas, una relación recurrente entre la complejidad del recorrido clínico, la institucionalización de las personas y la disponibilidad de la red sociofamiliar. Una trabajadora social refiere:

\footnotetext{
No tenía recursos internos o externos para afrontar situaciones, era muy impulsivo. Tenía un trabajo inestable, precario, que ponía su cuerpo en jaque. El paciente venía desesperado sin saber qué hacer y en lugar de explosionar, implosionaba y consumía. (Sonia, trabajadora social)
}

Asimismo, un psicólogo explica:

No se sabía de dónde venía, se había quedado en la calle, había tenido varios trabajos... había estado detenido, sentía cierto alivio por estar internado y la solución sería tener un lugar donde vivir, trabajo y no consumir más, estaba muy limitado a eso. (Manuel, psicólogo)

Se delimitaron dos líneas de análisis a partir de recurrencias en el discurso: por un lado, el pasaje sostenido y reiterado por dispositivos de salud mental como hospitales monovalentes ("psiquiatrizada") y comunidades terapéuticas ("impronta de acompañante"), así como también dispositivos carcelarios ("con antecedentes"). Por el otro, las reiteradas menciones a la red sociofamiliar como un faltante ("no tener a dónde ir", "familia que le hace el vacío", "desamparado", "errancia", "sin red").

Respecto a las consideraciones sobre los equipos profesionales intervinientes, en todos los casos se mencionó que a cada persona se le asignaba un equipo tratante, de composición interdisciplinaria con profesionales de trabajo social, psicología y psiquiatría. En seis entrevistas se hizo referencia a la "discusión del caso", "discusión de equipo", "objetivos del tratamiento", presencia o ausencia de "interdisciplina" lo que evidencia un horizonte de abordaje interdisciplinario como práctica institucional esperable.

Dentro del personal entrevistado, tres mencionaron que cada miembro del equipo tuvo una función diferenciada en la dirección del tratamiento y pudieron mencionar qué aspecto fue abordado por cada profesional:

\footnotetext{
Era un trabajo interdisciplinario, lo farmacológico tomaba la delantera porque era necesario para poder trabajar en otros espacios. En la terapia individual, trabajamos la relación con los hijos y el relato de un abuso sufrido a los 18 años. Con la trabajadora social se abordó por qué el padre cobraba su pensión y cuestiones relativas a su autonomía. (Paula, psicóloga)
}

Con relación a las consideraciones sobre las hipótesis diagnósticas es destacable que, en las entrevistas, seis profesionales mencionaron intervenir bajo la hipótesis diagnóstica de la psicosis. En términos generales, no hicieron referencia a una clasificación o marco teórico en particular, siendo "la psicosis" mencionada por profesionales tanto del área de psicología, trabajo social, como psiquiatría. La mitad del personal entrevistado mencionó la "dificultad" para delimitar el diagnóstico porque la persona "demanda poco", "no dice nada" o "está deteriorada".

El lugar que ocupa el consumo de sustancias en la subjetividad de las personas que asisten a los dispositivos apareció mencionado 
en la mayoría de las entrevistas al modo de un interrogante respecto a las coordenadas del consumo y a la función que cumple. En términos generales en las entrevistas no se desplegó un relato singular de la modalidad de consumo de cada persona y cómo éste se articula a la historia de vida. El consumo de sustancias apareció mayoritariamente asociado a situaciones de deterioro, excepto cuando tenía una función estabilizante. Las y los profesionales que hicieron mención a la función del tóxico, lo hicieron en términos relativos, como modo de apaciguar sintomatología psicótica:

El consumo la identifica como paciente, no se pudo correr de ahí, el consumo la define. (Bruno, psiquiatra)

Había algo del tóxico que le hacía cuerpo, era el efecto que le generaba. Ella, para mí, se empezaba a desestabilizar en su cuadro psiquiátrico y el hecho de consumir era como una automedicación, de calmar esos otros síntomas. (Julia, psicóloga)

\section{Dificultades y facilitadores en las trayectorias terapéuticas}

El segundo eje de la entrevista se centró en ubicar las dificultades y facilitadores que las y los profesionales de los equipos intervinientes evidenciaban en las trayectorias terapéuticas y cómo se abordaban dentro del tratamiento.

Respecto a los recursos con los que cuentan cada una de las personas tratadas, las y los profesionales destacan a la familia como un faltante: no tienen familia o se presenta de manera intermitente, o se considera un recurso "agotado por el riesgo". Solo en un caso se mencionó a la familia como una "red constituida", y en otro se consideró a un amigo como recurso.

No fueron mencionadas instituciones por fuera de la familia y el Estado en términos de derechos específicos: subsidio habitacional y pensión por discapacidad. En un solo caso se mencionó el trabajo como un recurso con el que cuenta la persona usuaria del dispositivo, mientras en el resto no se mencionó o se ubicó como faltante. Los recursos se presentan muchas veces como elementos aleatorios sin una lógica aparente como, por ejemplo: "información sobre un parador", "la escucha del padecimiento", un "taller de carpintería", la "medicación psiquiátrica", "agilización de subsidio para vivienda".

Con relación a los "recursos subjetivos" fueron nombrados como: el "armado débil", "lábil", "tenía pocos", "sin mucho capital simbólico o posibilidad de introspección", siendo considerados como un obstáculo al tratamiento.

Recursos subjetivos tenía pocos, no tenía mucho capital simbólico o posibilidad de introspección, estaba como anestesiada. (Manuel, psicólogo)

Solo en una entrevista una psiquiatra mencionó "el recurso subjetivo" como algo con lo que el usuario contaría:

El paciente tenía tela, podía pensar un montón de cosas, tenía cierta inteligencia [...] tenía cosas medio manipuladoras. (Marcos, psiquiatra)

Las y los profesionales ubican como algo deseable para el trabajo terapéutico la capacidad de simbolización y el registro de su situación por parte de las personas: "tener registro de lo que pasaba alrededor y estuviera en condiciones de decidir"; sin embargo, no mencionan estrategias concretas para el abordaje de estas dificultades. Se podría establecer que una concepción que subyace a los equipos de profesionales entrevistados es la necesidad de contar con lazos familiares y un capital simbólico considerable para llevar adelante un tratamiento.

Los principales obstáculos en las trayectorias terapéuticas fueron atribuidos a las personas usuarias de los dispositivos en la mayoría de las entrevistas: 
Era un paciente que no habla, no decía nada. Tenía pensamiento concreto, cierta debilidad. (Mauro, psiquiatra)

Las repeticiones del paciente, poca implicación subjetiva y dificultades para aceptar normas institucionales. (Paula, psicóloga)

Estos obstáculos parecerían entenderse como características o atributos de las personas atendidas, no como provenientes de la vinculación con un entorno sociocultural, estructura familiar o cualquier otra categoría relacional, histórica o sociocultural. En reiteradas oportunidades, el obstáculo se centró en la dificultad de construir una demanda de tratamiento subsumida, en algunos casos, a la ausencia de demanda por parte de las personas y, en otros, como una dificultad de lectura en los equipos intervinientes:

\section{¿Que venía a tratar al hospital? venía al hospital a conseguir una casa, un trabajo y dejar de drogarse: no había más que un contexto vulnerable pero no en urgencia, nunca lo vi muy enganchado con el tratamiento. (Manuel, psicólogo)}

En diferentes entrevistas los obstáculos principales fueron ubicados del lado de la oferta de tratamiento: la escasez de turnos para derivar, diferencias terapéuticas entre el equipo tratante, poca coincidencia de horarios entre profesionales del mismo equipo y cambios institucionales. Resultó significativa la falta de articulación en las derivaciones intrahospitalarias o su escasa mención, considerando las características de los casos clínicos relevados. Incluso, en una entrevista nombraron como derivación el hecho de conseguir turnos con el equipo interdisciplinario de un nuevo dispositivo intrahospitalario, sin que eso se acompañe de un intercambio entre los profesionales intervinientes.

Con relación a las derivaciones hacia otras instituciones, en un solo caso se observó que la indicación terapéutica consistió en la derivación a otro efector de salud y vivienda, que se realizó acompañando el proceso de derivación de forma articulada con el efector de salud, y ambos coincidieron en la forma de abordaje:

...necesitaba un lugar donde externarse con una continuidad de cuidados. (Melisa, trabajadora social)

...lo derivamos a un hogar para personas portadoras de VIH, estuvimos tres meses con llamados telefónicos y entrevistas con la gente de la institución, fuimos con él y seguimos el tratamiento allá porque no tenían profesionales, una vez por semana, luego cada 15 días. (Ivana, psicóloga)

Las interrupciones en el tratamiento fueron mencionadas de manera heterogénea por las y los profesionales entrevistados como: "revocación", "separación", "abandono", "externación". En uno de los casos se nombró la interrupción del tratamiento como "separación" por infringir normas institucionales (consumo y situaciones de violencia) en el contexto de la internación.

Del personal de salud entrevistado, tres presentaron una lectura explicativa respecto al abandono del tratamiento en términos clínicos de orientación psicoanalítica como "un acting out dirigido a la madre" o "un deseo de presentarse, acá estoy, mirame". En el resto de los casos, en los que se menciona el abandono del tratamiento, los equipos entrevistados no recordaron las coordenadas.

Los resultados obtenidos en este eje coinciden, en parte, con algunas conclusiones publicadas en la investigación llevada adelante por la Asociación Civil Intercambios en la Ciudad de Rosario:

Los trabajadores criticaron las creencias, prácticas y valores que los sujetos tienen sobre su propia salud y, en gran medida, señalaron que esta dimensión actúa como una barrera en el acceso, se tiende en gran medida a una individualización del problema del acceso o a la reducción del problema de accesibilidad a problemas del propio sujeto, mientras que las 
cuestiones de tipo organizacional, por ejemplo, no aparecen como una barrera importante. ${ }^{(1)}$

\section{Estrategias terapéuticas}

El tercer eje de las entrevistas abordó las estrategias terapéuticas que los equipos intervinientes llevaron adelante en función de la conceptualización del caso, de los obstáculos y los facilitadores delimitados en los recorridos clínicos de las personas.

La lógica de la prescripción en salud apareció sosteniendo los discursos de las y los profesionales. En lo relativo a los objetivos terapéuticos, la delimitación pareciera ser una tarea exclusiva de los equipos intervinientes y no un trabajo en conjunto con las personas que asistían al dispositivo. La misma lógica se evidenció con las indicaciones terapéuticas, especialmente en lo relativo al manejo psicofarmacológico y de las derivaciones:

Seguía bastante las indicaciones, eso era algo a favor. Estaba muy preservada la relación con los profesionales, podía escuchar lo que se le iba señalando. (Sonia, trabajadora social)

El paciente aceptó porque ya no tenía adónde ir. (Manuel, psicólogo)

Ubicamos tres vertientes centrales en las estrategias terapéuticas: el trabajo sobre la adherencia, las maniobras orientadas a acotar o disminuir el consumo de sustancias y los modos de abordaje de las cuestiones clínicas. Respecto de la adherencia, relevamos dos ideas distintas en función de si la misma era ubicada como obstáculo o como estrategia en pos de una oferta en salud. Al hablar de la falta de adherencia asociada al obstáculo en el tratamiento, se observó una tendencia a depositar en las personas la responsabilidad de cumplir con el "deber ser" del tratamiento $^{(8)}$. En cambio, la adherencia ubicada del lado de la estrategia, fue nombrada como el intento de "alojar" subjetivamente a la persona que demanda, o la construcción de una referencia a la institución hospitalaria como un lugar al que acudir frente a situaciones de urgencias subjetivas, aumento del consumo de sustancias o riesgo para sí o terceros.

Las prácticas de autocuidado y pautas de alarma con relación al consumo problemático de sustancias aparecieron en las entrevistas más asociadas a una referencia teórica e institucional que a una estrategia delimitada de abordaje. Podemos pensar que la perspectiva de reducción de riesgos y daños se encuentra presente en el abordaje institucional, aunque de manera naturalizada por las y los profesionales. Por ejemplo, se hace mención a la idea de regular o acotar el consumo de sustancias como un objetivo terapéutico a trabajar, pero no dan cuenta de que el umbral mínimo de exigencia que utilizan como encuadre constituye una estrategia terapéutica. Tampoco se utilizan términos como abstinencia, recaídas o sanción al consumo, en cambio, sí se hace referencia al lugar que ocupa el tóxico en la subjetividad de las personas que asisten a los dispositivos. En un solo caso, una psicóloga hizo alusión a una intervención en el marco del autocuidado y la reducción de riesgo:

\section{...yo había problematizado con ella el consumo en el Riachuelo. Se puede con- sumir en otro lado, como un intento de reducir daños. (Paula, psicóloga)}

En términos generales, el autocuidado registrado por las y los profesionales fue aquel vinculado a la responsabilidad de las personas frente a sus enfermedades clínicas, especialmente en personas con $\mathrm{VIH}$ :
Tenía consumo de varias sustancias, pero cuando empezaba a inyectarse [cocaína] era una alarma para ella. Ella llevaba muy bien su tratamiento de HIV y de repente eso empezaba a tambalear, no iba a la infectóloga, ahí fue el lugar de la internación. (Julia, psicóloga)

El autocuidado se transforma aquí en un indicador de la conducta irracional de las personas ${ }^{(15)}$, que abona incluso a la indicación de una internación en salud mental. Solo en 
una entrevista se contempló la práctica de autocuidado con relación al desarrollo de la autonomía personal.

...se trabajaron cuestiones relativas a la autonomía, el padre cobraba la pensión, y fuimos armando un camino para que la cobre ella. (Melisa, trabajadora social)

Al interrogar sobre los objetivos y las estrategias terapéuticas nos encontramos con cierto impedimento por parte de los equipos de profesionales para responder a las preguntas. El quehacer profesional parece presentar una dificultad para ser nombrado. A pesar de esto, en las historias clínicas aparecen explicitadas algunas estrategias, que las y los profesionales no ubican como tales como, por ejemplo, la estrategia de flexibilización del encuadre utilizada en algunos casos para alojar a las personas en pos de sostener los tratamientos. Esta dificultad abrió un interrogante acerca de cómo se delimitan y registran las prácticas profesionales y cómo son percibidas por las y los profesionales que trabajan en salud mental. Existen informes que dan cuenta del subregistro de las prestaciones profesionales mayoritariamente asociado a que la complejidad de las temáticas abordadas deriva en una falta de acuerdo respecto a su codificación. Otra cuestión que se asocia al subregistro es la naturalización de las prácticas por parte del personal de salud como, por ejemplo, aquella mencionada sobre la perspectiva de reducción de riesgos y daños ${ }^{(16)}$.

Indagamos sobre el horizonte del alta para este perfil de personas. Las respuestas han sido diversas $y$, aunque desde marcos referenciales heterogéneos, se observó la dificultad para imaginar un posible alta. Esta dificultad era atribuida a las características de la persona atendida, a las dificultades del trabajo interdisciplinario y a las instituciones:

Sin una dirección u objetivo interdisciplinario de tratamiento no hay un final. Al no pensar adónde vamos nunca termina y va y viene. (Ivana, trabajadora social)
El rumbo errático es de las instituciones no del paciente. (Marcos, psiquiatra)

Resultó llamativo que las y los profesionales no hablaran de padecimiento crónico. Algunas investigaciones sobre la temática ubican que la enfermedad crónica ha interpelado los modos de nombrar aquello que es esperable en un tratamiento y que en lugar de hablar de curación se habla de procesos, mantenimientos y cuidados ${ }^{(17)}$.

\section{Tramos, trayectorias y equipos intervinientes}

Este apartado da cuenta de un resultado que no tuvo que ver con lo relevado en las entrevistas, sino con un momento previo, en el que relevamos los equipos profesionales intervinientes en cada recorrido clínico para definir a quiénes entrevistar. Los resultados de dicho relevamiento fueron muy heterogéneos entre sí. En todos los casos los recorridos clínicos están conformados por más de dos trayectorias, siendo el mínimo dos y el máximo 23.

De este modo, se desprende la participación de un número alto de profesionales intervinientes en cada recorrido clínico. Se relevó que estos oscilan de cinco a 21 en cada recorrido clínico. Cabe destacar, que este número depende del tiempo que la persona esté realizando algún tratamiento en el hospital. Estos extremos los representan Romina, quien comenzó a realizar tratamiento en el año 2016 y tuvo cinco profesionales intervinientes y Clara, quien comenzó a realizar tratamiento en el año 2006 y tuvo 21 profesionales intervinientes. Podemos inferir a partir de los datos relevados que, a mayor cantidad de tiempo en la institución, mayor cantidad de profesionales intervinientes en el recorrido clínico.

A partir de identificar la cantidad de trayectorias de cada recorrido clínico, nos encontramos con la necesidad de incorporar una herramienta nueva de análisis. Relevamos que, en el $80 \%$ de los casos, las trayectorias contaban con subpartes, delimitadas por 
las derivaciones intrahospitalarias entre los dispositivos de consultorios externos, hospital de día, internación e internación aguda. Decidimos nombrar tramos a estos momentos, que presentan tanto diferentes dispositivos de atención como equipos intervinientes dentro de una misma trayectoria.

El concepto de tramo al que debimos apelar mientras recolectamos los primeros datos, problematiza la definición misma de recorrido clínico errático que habíamos formulado en la investigación previa. El tramo viene a dar cuenta de un momento en el que el tratamiento se sostiene, aunque implique el tránsito entre dispositivos.

Esto se evidencia, en el caso de Clara, nombrada por el mayor número de dispositivos en la primera investigación (internación, internación aguda, atención a la demanda espontánea, consultorios externos). Fue quien más profesionales intervinientes tuvo: 21 . Su recorrido clínico presenta dos trayectorias: la primera finaliza con una derivación a un hospital monovalente de salud mental de la Ciudad Autónoma de Buenos Aires, que la usuaria acepta. Regresa re-derivada cuatro años después, comenzando su segunda trayectoria en el hospital. Esta última cuenta con doce tramos (Tabla 1). Siempre ha aceptado y sostenido las indicaciones terapéuticas, transcurriendo por diferentes dispositivos del hospital.

En este punto nos encontramos con una tensión epistemológica entre la salud colectiva y el modelo biomédico a la hora de describir y analizar los tratamientos de las personas. Tratamiento es un concepto propio del modelo biomédico, que tiene una concepción prescriptiva, en la que quien conduce es el profesional de la salud. En cambio, dentro de la salud colectiva se habla de itinerarios terapéuticos, donde la participación de las personas usuarias de los dispositivos es fundamental. El concepto de tramo, habita esta tensión ya que está presente una lógica preceptiva por parte de las y los profesionales en la dirección del tratamiento que implica una derivación a otro dispositivo, pero también la decisión de cada persona de continuar o no con el tratamiento.
Creemos que el concepto de tramo está vinculado al de adherencia, pero principalmente al concepto de trato que propone Epele $^{(18)}$. La autora analiza la creación de nuevos tratamientos frente a los nuevos problemas que presentan las condiciones de emergencia, que terminan mostrando un carácter de montaje entre diversos modos de tratar expertos y legos. Definimos el trato y los modos de tratar desde los aportes de Ulloa ${ }^{(19)}$ como la descripción y clasificación de las acciones orientadas hacia otros y de los otros respecto a sí, que construyen patrones con valoraciones morales: maltrato, destrato y buen trato.

Como postula Epele ${ }^{(18)}$, "la mirada de los tratamientos desde la noción del trato permite incluir en el análisis y categorizar la materia de la que están hechos los vínculos que nos hacen y deshacen". Sostenemos que algo del buen trato se ha puesto en juego en estos tramos que permiten una permanencia y posibilitan un tratamiento. Como explica Ulloa ${ }^{(19)}$, el buen trato está vinculado con la ternura e incluye, de este modo, algo de la intuición, del miramiento y de la donación simbólica, produciendo un tratamiento adecuado, que sostiene al otro como sujeto, lo reconoce desde su singularidad y genera así diversas formas de autonomía.

Prácticas vinculares como los tratamientos pueden entenderse como técnicas y tácticas que contienen un saber no formulado explícitamente, pero que vienen participando en el abordaje ${ }^{(18)}$. Esto se evidenció en la investigación, dado que no se mencionó en ningún caso la existencia de otros equipos intervinientes en el recorrido clínico de las personas, siendo que en todos los casos hubo presencia de un número alto de profesionales intervinientes. Es decir, no se identificaban estos tramos como momentos dentro de las trayectorias, en los que algo del tratamiento tenía efectos.

\section{DISCUSIÓN}

Esta investigación intentó describir la perspectiva de las y los profesionales intervinientes 
respecto a los recorridos clínicos de personas con consumos problemático de sustancias en un hospital nacional monovalente de la Ciudad Autónoma de Buenos Aires, especializado en salud mental y adicciones. Se basó en un estudio previo en el que el recorrido clínico errático se definió como: "usuarios que establecen con el hospital un vínculo discontinuo y errático, que inician el tratamiento, lo interrumpen, volviendo a consultar en reiteradas ocasiones y transitan por diferentes dispositivos dentro del hospital". Esta definición resultó poco descriptiva del recorrido de algunos casos, por lo que para esta investigación se apeló a un nuevo concepto: el tramo. Este nuevo concepto vino a dar cuenta de un momento en el que la trayectoria se sostiene, aunque implique el pasaje por un dispositivo diferente o el tránsito entre dispositivos. Tal el caso de Clara, nombrada en el relevamiento de la muestra inicial por el mayor número de dispositivos, cuya segunda trayectoria en el hospital cuenta con doce tramos y quien siempre ha aceptado y sostenido las indicaciones terapéuticas, transcurriendo por diferentes dispositivos del hospital. Esta situación nos llevó a interrogar la propia noción de lo "errático", en tanto los movimientos en la trayectoria de Clara responden a indicaciones por parte de los equipos intervinientes, no a discontinuidades o interrupciones de la usuaria.

Resultó interesante el contraste entre la pregnancia de lo "errático" como modo de describir a las personas usuarias de los dispositivos y las lecturas clínicas realizadas en los momentos de interrupción de cada recorrido clínico. Respecto de estos se relevó en la mayoría de las entrevistas una ausencia de hipótesis clínicas sobre las discontinuaciones de las personas.

Se desprende de esta investigación la importancia de repensar si lo que institucionalmente se nombra como "errático" es un obstáculo en los tratamientos o si, por el contrario, es preciso articular las discontinuidades como aspectos característicos de los itinerarios terapéuticos en general y de los recorridos clínicos en particular, de personas con padecimientos crónicos. Incluso, Ilama la atención la ausencia en las entrevistas realizadas del concepto de padecimiento crónico en salud mental. Sería una temática interesante para abordar en futuras investigaciones.

Las y los profesionales definieron, en líneas generales, dos series de obstáculos en los tratamientos asociados a las características de las personas: la "falta de recursos subjetivos" y la falta de recursos sociales, entendemos ambos insertos en una trama de ciudadanía: trabajo, vivienda, participación activa en la comunidad, formación y red de vínculos. Resultaría interesante ahondar en estas consideraciones en futuras investigaciones para indagar las representaciones de los profesionales tienen en torno al "trabajo con la familia", para hacer lugar a líneas de intervención en aspectos que se consideran relevantes por su recurrencia por parte de los equipos intervinientes en tanto obstáculo: para dar un alta de internación, para el sostenimiento de un tratamiento o la estabilidad de una presentación clínica.

En otras palabras, la investigación llevada adelante por Intercambios, Ilega a conclusiones similares:

...se mencionaron principalmente barreras culturales (prácticas, hábitos, creencias y valoraciones de los usuarios de drogas) entre las que se destacan los comportamientos relacionados con la práctica de consumo de drogas. Otros motivos mencionados fueron las situaciones laborales y los problemas con las redes cercanas (familiares, pareja, amigos). Por último, se refirieron motivos incluidos en barreras económicas y geográficas [...] la mayoría de las respuestas dadas por los trabajadores adjudican los motivos de la interrupción a aspectos culturales que residen básicamente en el sujeto y refieren a las prácticas y las valoraciones de los pacientes acerca de su salud. Este tipo de argumentación se presenta como la principal barrera. [...] Estas interpretaciones ponen de manifiesto las representaciones sociales de los trabajadores sobre los UDs [usuarios de drogas], argumentando que la barrera de acceso de tipo cultural se relacionaba con "el 
estilo de vida" del sujeto, la no preocupación por su salud y/o el "olvido" de las citas pautadas aduciendo, por ejemplo, que no concurrían a las consultas "por estar drogados". ${ }^{(1)}$

Con relación a los objetivos y las estrategias terapéuticas, se relevó una notoria dificultad tanto para nombrarlos como para delimitarlos. Nos resulta paradigmático el uso de una estrategia terapéutica que no fue explicitada: "la flexibilización del encuadre". Esta herramienta de intervención no fue mencionada por los equipos de profesionales entrevistados a pesar de que sí se había dejado constancia escrita en las historias clínicas. Esta diferencia abre un interrogante respecto de cómo se conceptualizan las intervenciones y si el equipo tratante las percibe como tales. De este modo, las y los profesionales parecieran adoptar una posición amigable con relación a los encuentros y desencuentros entre las personas y la institución y pensar en las condiciones que hacen posible el sostenimiento de una trayectoria, sin Ilegar a conceptualizarlo como una modalidad de intervención.
Nos encontramos con modalidades de trayectorias terapéuticas diferentes, que no responden al tipo de pacientes "adherente" o "no adherente" sino que conjugan las condiciones de vida de personas con la experiencia subjetiva y singular del proceso salud-enfermedad ${ }^{(15)}$.

Finalmente nos resultó Ilamativo, por su recurrencia, la poca sistematización de las derivaciones intrahospitalarias entre equipos tratantes de salud mental, en comparación a las interconsultas médicas clínicas y derivaciones a otros efectores de salud que adquieren un carácter asistido. A su vez, se relevó la falta de comunicación entre equipos intervinientes de diferentes tramos de un mismo trayecto, aunque ello no es nombrado como dificultad u obstáculo en un tratamiento.

A los fines de nuestra investigación consideramos que esta característica de la comunicación institucional puede influir en la lectura que los equipos profesionales intervinientes tienen de determinados casos: una imagen parcial y fracturada-circunscripta a un tramo- que no se consigue estructurar en una imagen integradora que dé visión de conjunto al recorrido clínico de las personas usuarias de los dispositivos.

\section{AGRADECIMIENTOS}

Para la presente investigación contamos con el apoyo del Área de Investigación del Hospital Nacional de Salud Mental y Adicciones Lic. Laura Bonaparte, para la cual recibimos una beca durante el período 2017-2018. Queremos agradecer especialmente a Silvia Gianni, por su acompañamiento y guía, y a las y los profesionales que participaron de las entrevistas, que desinteresadamente nos brindaron su tiempo.

\section{REFERENCIAS BIBLIOGRÁFICAS}

1. Rossi D, Pawlowicz MP, Zunino Singh D. Accesibilidad de los usuarios de drogas a los servicios públicos de salud en las ciudades de Buenos Aires y Rosario: la perspectiva de los trabajadores de la salud. Buenos Aires: Intercambios Asociación Civil; 2007.

2. Lewkowicz I. Subjetividad adictiva, un tipo psicosocial instituido: Condiciones históricas de posibilidad. En: Dobon J, Hurtado G, (comp.). Las drogas en el siglo... ¿qué viene?... Buenos Aires: Ediciones FAC; 1999. p. 91-107.
3. Romani O. Adicciones, drogodependencias y "problema de la droga" en España: la construcción de un problema social. Cuicuilco. 2010;17(49):83-101.

4. Romani O. Criterios de prevención: un debate necesario. En: Hopenhayn M, (comp.). Prevenir en drogas: enfoques integrales y contextos culturales para alimentar buenas prácticas. Santiago de Chile: Naciones Unidas; 1999.

5. Stolkiner A. Nuevos enfoques en Salud Mental [Internet]. Buenos Aires: $14^{\circ}$ Congreso Latinoamericano de Arquitectura e Ingeniería Hospitalaria; 2003 [citado 20 jun 2020]. Disponible en: https://tinyurl.com/ycyagtza.

6. Stolkiner A. Interdisciplina y Salud Mental [Internet]. IX Jornadas Nacionales de Salud Mental, I Jornadas Provinciales de Psicología: Salud mental y mundialización, estrategias posibles en la argentina de hoy; Posadas, Misiones, 2005 [citado 10 ago 2019]. Disponible en: https://tinyurl.com/ybx22js2.

7. Almeida-Filho N, Silva Paim J. La crisis de la salud pública y el movimiento de Salud Colectiva en Latinoamérica. Cuadernos Médico-Sociales. 1999;(75):5-30. 
8. Margulies S. La adherencia a los tratamientos: un desafío para la atención del $\mathrm{VIH} / \mathrm{sida}$, una lectura desde la antropología. Actualizaciones en Sida. 2010;18(68):63-69.

9. Comes Y, Solitario R, Garbus P, Mauro M, Czerniecki S, Vázquez A, Sotelo R, Stolkiner A. El concepto de accesibilidad: la perspectiva relacional entre población y servicios. Anuario de Investigaciones. 2007;14:201-209.

10. Cabral ALLV, Martinez-Hemáez A, Andrade EIG, Cherchiglia ML. Itinerários terapêuticos: o estado da arte da produção científica no Brasil en Ciência \& Saúde Coletiva. 2011;16(11):4433-4442. doi: 10.1590/S141381232011001200016.

11. Menéndez E. Modelos de atención de los padecimientos: de exclusiones teóricas y articulaciones prácticas. Ciência \& Saúde Coletiva. 2003;8(1):185-207.

12. Argentina, Salud Pública. Ley 26.657: Ley Nacional de Salud Mental [Internet]. 2010 [citado 10 ago 2019]. Disponible en: https://tinyurl.com/zl7bzbl.

13. Argentina, Salud Pública. Decreto 603/2013: Ley 26.657 [Internet]. 2013 [citado 10 ago 2019]. Disponible en: https://tinyurl.com/y6wod984.
14. Minayo MCS. La artesanía de la investigación cualitativa. Buenos Aires: Lugar Editorial; 2009.

15. Margulies S, Barber N, Recoder ML. VIH-sida y “adherencia" al tratamiento: Enfoques y perspectivas. Antípoda. 2006;(3):281-300.

16. Dirección Nacional de Salud Mental y Adicciones. Experiencias de sistematización de información en salud mental y adicciones [Internet]. 2012 [citado 10 ago 2019]. Disponible en: https://tinyurl.com/yala4mch.

17. Del Mónaco R. Autocuidado, adherencia e incertidumbre: tratamientos biomédicos y experiencias de pacientes en el dolor crónico de la migraña. Salud Colectiva. 2013;9(1):65-78. doi: 10.18294/sc.2013.201.

18. Epele M. El tratamiento como palimpsesto: Cuando la medicalización se convierte en crítica "políticamente correcta". Cuadernos de Antropología Social. 2013;(38):7-31.

19. Ulloa F. Novela clínica psicoanalítica: Historial de una práctica. Buenos Aires: Libros del Zorzal; 2012.

\section{FORMA DE CITAR}

Alli A, Minond J, Embil Y, Kiss Rouan M. Modos discontinuos y erráticos de transitar un hospital especializado en salud mental y adicciones de la Ciudad de Buenos Aires: la mirada de profesionales de la salud. Salud Colectiva. 2020;16:e2521. doi: 10.18294/sc.2020.2521.

Recibido: 31 ago 2019 | Versión final: 9 jun 2020 | Aprobado: 16 jun 2020 | Publicado en línea: 14 jul 2020

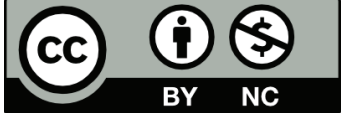

Esta obra está bajo una licencia de Creative Commons Reconocimiento-NoComercial 4.0 Internacional. Reconocimiento - Permite copiar, distribuir y comunicar públicamente la obra. A cambio, se debe reconocer y citar al autor original. No Comercial - Esta obra no puede ser utilizada con finalidades comerciales, a menos que se obtenga el permiso. 